\title{
STRUCTURAL-SEMANTIC AND TEXTUAL PARAMETERS \\ OF PROFESSIONAL FITNESS LANGUAGE
}

\author{
Ivanna Melnychuk \\ Lesya Ukrainka Eastern European University, \\ Lutsk, Ukraine \\ yana19mel@gmail.com \\ Iryna Navrotska \\ Lesya Ukrainka Eastern European National University, \\ Lutsk, Ukraine \\ rasmusia@ukr.net
}

Received March, 19, 2020; Accepted May, 07, 2020

\begin{abstract}
The article is devoted to a comprehensive study of the structural, semantic and textual characteristics of the English professional language of fitness. Achieving the goal involves the following tasks: exploring the stages of development and genesis of a professional language of fitness; distinguishing its terminology; analysing the structural features of terminological fitness vocabulary in order to identify productive ways of creating lexical units; understanding the nature of lexical-semantic relations in the professional language of fitness; identifying the terminological frequency and richness of the professional text. These tasks are realised throuth the implementation of the logical text analysis of Instagram blogs, definition analysis (etymological and wordbuilding) and componential analysis. The modern world is characterised by the dynamic development of new scientific and professional fields, which have an ostensible impact on the society's level of social and cultural life. Taking into account these processes and phenomena, terminological studies of professional languages acquire special significance, allowing to reveal the leading development characteristics and factors of various linguistic-professional spheres. The theoretical significance of the article pertaining to the multidimensional analysis of the English professional language of fitness enriches and extends the theoretical foundations of terminology, specialised studies of languages, lexicology and lexicography. The study results testify to the fact that fitness terminology is a complex notion comprising the borrowed and newly coined units. The higher professional level of a text is usually characterised by more sophisticated sematic choices. The findings are practically important for the further study of the formation and functioning mechanisms of other professional languages.

Key words: professional fitness language, structural and semantic features of Instagram fitness blogs, textual characteristics of fitness professional texts.
\end{abstract}




\section{Introduction}

The development of the contemporary fitness culture and industry is the aftermath of complex historical processes. The transformation and globalisation of gym and fitness culture is structured in three different but partly overlapping phases of development. This global enterprise transforms mainly through the structural transformations of society and the cultural and social processes of mediatisation, and individualisation.

Today, professional fitness blogs and gyms are a huge global business. Fitness has become the overall concept used when referring to health clubs, fitness franchises, and fitness gyms. Fitness thereby has turned into a folk movement, but not one comparable to the old 20th-century movements, often connected to national sentiments, but a highly individualised and personal task instead. The blurring of the relation between health and beauty, although manifested in slightly different ways nationally, is a central part of this transformation.

The face of fitness gyms has changed, and it is possible to talk about a fitness revolution.

The development of the fitness industry is to a great extent an international and global history. The techniques, tools, and physical exercises used today in gyms all over the world are the results of a physical culture developed and refined during the 20th century. This culture also has roots stretching back to ancient civilisations. Some examples:

- Asia: The teachings of Confucius encouraged the participation in physical activity to prevent certain diseases associated with inactivity like organ malfunctions and internal stoppages. This led to the development of Cong Fu gymnastics, a series of stances and movements to promote circulation and keep the body in good working order.

- Greece: The ancient Greeks focused on the idealism of physical perfection and an importance on fitness unparalleled in history. The development of a strong body was as important and essential for the development of a strong mind.

- Rome/Persia: The ancient Roman and Persian societies both implemented empire-wide training programs recognising the relationship between physical fitness and performance on the battle field. While both empires are known for their success in conquering and expansion as a result, ironically, the downfall of both came from becoming so big and affluent that physical activity declined and they the history of gym culture, the body ideals, exercises, techniques, and the pedagogy of fitness have become an increasingly international enterprise.

With the industrial age humming along, fitness continually reappears as a fashion depending on political circumstances. The periods leading up to and during World Wars cause a focus on national fitness levels, while post-war periods placed emphasis on leisure.

In spite of all the science and more money than ever being spent on fitness, there is an obesity epidemic and the future looks worse. In other words, regardless of the problem concerned with a specific aspect of life, the general fitness, and consequently, the health of the population continues to decline. Simultaneously, the fitness industry is changing, with less emphasis on fancy toys which cannot help to lose weight, for example: smart fitness belt, sauna suits, Tom Brady's TB12 
vibrating foam roller and more emphasis on functional training.

For consumers, there is a huge surge in fitness devices, instagram blogs, tracking apps and other tools to quantify what is going on inside, while at the industry level there is an emergence of more smaller, gym facilities.

The key to this is using science and technology to empower fitness professionals to be more effective and accessible.

\section{Formulation of the research problem and its significance}

Today in modern linguistics there are several equivalent terms for the concept of "professional language" - "language for special purposes", "subtext", "sublanguage" and others. In British and American linguistics, the term "language for special purposes" (LFP) is used to denote professionally marked vocabulary. German linguist Lothar Hoffmann uses the term "professional language". L. Hoffmann defines professional language as "a set of all language tools used in a specially defined communicative field in order to achieve understanding between all professionals in a particular field" [1, p. 53].

By a set of linguistic means L. Hoffmann understands not only phonetic, morphological and lexical elements and rules of syntax, but also their functional interaction in any possible acts of communication in this area. He divided all language means into three classes:

1. language means, which are available in all sublanguages;

2. language tools that are available in all professional languages;

3. language tools that are available in only one professional language.

Terminological vocabulary, the number of which is constantly growing, occupies an important place in the vocabulary of the language. This vocabulary group includes all words that are combined in the language under the common name "terms". The term is a special word or phrase that is used to accurately express the concept of a particular field of knowledge - science, technology, socio-political life, art, economics and more. When selecting terms for the professional language of fitness, it is necessary to include those meanings of the word that express the concept of the industry. It should be noted that the vocabulary of the professional text can be divided into types:

- terms of a particular industry or inter-industry terms;

- intersectoral terms (terminological units of related sciences);

- half-terms, which include nomenclature;

- professional jargon, which does not claim to be accurate and unambiguous.

Fitness is now actively developing, and its relatively recently formed terminology, which includes terms from related fields of anatomy, biomechanics, biochemistry, sports physiology, anthropometry, etc., requires scientific understanding.

In the early stages of the study of professional languages, linguists L. Hoffmann and R.G. Piotrowski considered the language of professional communication as a subtext of science and technology.

Professional communication is a language communication, with all the characteristic features and is filled with professional terms. Communication involves primarily communicative management, so the participants in the process of communication are connection between the communicant and the surrounding reality. 
The professional language of fitness is a relatively young professional language consisting of a set of all language tools used in professional communication. The vocabulary of fitness is meant as a set of tokens used in professional communication of common language, terms of sports, medicine, nutrition, fitness and general scientific terminology, as well as nomenclature units and professionalisms. This composition of fitness vocabulary is due to the fact that professional communication takes place not only at the professional level, but also between ordinary people who want to improve the quality of life and well-being, and therefore it must meet a wide range of communicative needs and requirements.

\section{Research Methods}

The study is based on the examination of professional fitness Instagram blogs. The purpose of the article is to identify, describe and summarise the structuralsemantic and textual features of the English professional language of fitness. The material of the research is professional texts in the field of fitness, as well as terms of fitness. The source of the material was professional publications, terminological dictionaries, articles in professional blogs that cover the problems of fitness.

The main approaches of the dialectic of scientific cognition became the methodological basis of the research: systemic, diachronic, structural-synchronic and functional.

The analysis of structural and semantic characteristics of the English professional language of fitness is carried out on the basis of etymological and structural definitions, as well as means of logical analysis. Semantic, word-forming and componential analyses allow to investigate the composition of the fitness terminology, to present it systematically, as well as to analyse the lexical and semantic features of terms on the material of Instagram blogs.

\section{Theoretical provisions of the study of professional languages}

In linguistics, there is no single overview of the definition of "professional language". Until the 1970s, the interpretation of this term was limited to studies of terminology in the field of fixation and standardisation. With the development of terminology, the term "professional language" began to cover the morphological and syntactic and stylistic elements of special texts, as well as their structural characteristics.

Theoretical and methodological foundations of the study of professional languages were formed in the works of linguists L. Hoffmann, G.F. Fluka, W. von Hahn, K.-D. Bauman.

L. Hoffmann defines professional language as "the totality of all language tools used in the professionally closed sphere of communication in order to ensure understanding between people working in this field" [2 p. 139]. T. Kyiak believes: "to this definition should be added the fact that the functioning of the professional language provides only clearly defined terminology". The basis of the study are professional texts, namely the semantic core - the terms of the field, because the most important means of expressing professional language is a professional text that describes the real objects of the subsystem, reflects the real picture of its terminology". 
The well-known terminologist $\mathrm{H}$. Felber interprets the definition of the term as follows: "A term is a conditional symbol (word, group of words ...), which expresses a certain concept in a certain field of knowledge" [4, p. 54].

L. S. Lotte gives the following interpretation: "A term is a lexical unit of a certain language for special purposes, denoting a general, specific or abstract concept of the theory of a certain special field of knowledge or activity". Terms are elements of terminological systems that reflect theories that describe special areas objects of language for special purposes. The variety of definitions of the term "term" is primarily due to the lack of clearly defined characteristics, as a result of which each scientist understands this phenomenon in his own way.

Thus, professional language exists on the basis of commonly used language as a means of professional communication of specialists, and professional text is the main representative of professional communication, which provides an opportunity to fully disclose its features. The semantic core of any professional language is a term - a linguistic sign that correlates with a certain concept of professional language and enters into a systemic relationship with other words within a closed system.

The main requirements for the term are its systematisation, the presence of definition, accuracy, brevity and unambiguity. All terms are related to certain terminology and are elements of certain terminological systems. Like any lexical unit, the term performs a number of functions in professional communication: nominative for naming certain concepts, objects, phenomena; significant to denote a general concept, which is an element of a system of special concepts of a particular branch of science or technology; cognitive, which defines the term as the result of a long process of learning the essence of objects and phenomena of objective activity and inner life of man, as the verbalisation of a special concept (V. Leichik); communicative for the transfer in time and space of professional knowledge (V. Leichik) [6].

The professional language of fitness is one of the young professional languages consisting of a set of all language tools used in professional communication. Nomenclature units and professionalisms play a significant role in professional communication, because communication between specialists can take place at different levels. Despite its importance, they are not part of the terminology. In contrast to terms, nomen (for example, George's system - система Джорджа) is characterised by specific semantics and lack of definitions; they are opposed to terms, because their meaning is denotative, objective. Professionalisms (for example, toning тонізувати своє тіло і набути форми) differ from the terms by non-normative use and stylistic labelling; they are professionally spoken doublets of officially recognised terms and are often formed by reducing terminological phrases and words.

Three main periods of development of the professional language of fitness have been recorded: 1) pre-scientific; 2) protoscientific or early scientific; 3 ) scientific. The first period is primary and is characterised by the borrowing of commonly used words into the terminology. Protoscientific, guided by special concepts that are mostly borrowed from Latin and ancient Greek, for example (competition, exercise, nutrition, obesity, gluteus). The last period is scientific, characterised by the emergence of narrow industry terms, for example (circuit training, tabata, kickboxing, spin class, general warm-up).

Nowadays the majority of people worry about their health and generally try to ensure their body is in good shape. People talk about fitness and work out routines on a day to day basis with using vocabulary of fitness he is useful to you talk 
professionally about your level of fitness or your exercise routine. So, the vocabulary of fitness is a set of tokens used in professionally communication of common language, terms of sports, nutrition, fitness, medicine and general scientific terminology, and also nomenclature units and professionalisms. This composition of fitness vocabulary is due to the fact that professional communication takes place not only at the level of professionals, but also between ordinary people who want to improve the quality of life and well-being, and therefore it must meet a wide range of communication needs and requirements.

\section{Structural-semantic parameters of professional fitness language}

The concept of «fitness» has become so comprehensive that it has acquired, in addition to a purely professional interpretation, also social and philosophical significance. From a philosophical point of view, fitness can be described as a system of balance of physical, mental, social and intellectual state of modern man, who lives in harmony with the environment.

Yes awareness of fitness brings its followers closer to the most dreamed and idealised times of Hellenistic harmony of the human personality.

Pragmatic or socially oriented understanding of the concept of fitness (from the English "to be fit" - to be healthy, be in shape) is a system of general health through certain physical activities, such as strength and aerobic training, flexibility, etc. in combination with a balanced diet.

Theorists of physical education and sports together with specialists in health training believe that the concept of "fitness" is based on the principle of FITT. This abbreviation is formed from the merging of four keywords: "frequency" (F) "frequency", is the number of classes per week; "Intensity" (I) - "intensity, tension", which is a characteristic of the severity of the load and is determined by the level of heart rate (HR); "Time" (T) is the time that presupposes the duration of classes, and finally, "type" (T) is the type or type of classes according to the means and methods used.

The terminological system of fitness shows the state of the science of fitness, sports and health programmes, as well as the study of their impact on general wellbeing. Conventionally, the fitness system can be divided into the following main points: the first - sports, the second - medicine and last but not least - is nutrition, which are divided into smaller sub-items.

Within the terminology of fitness, nine terminological microfields have been identified: «Fitness and Exercise Physiology», «Sports and Recreational Activities», «Physical activity and disease», «Training Methods and Equipment», «Body Structures and Movements», «Disorders and Diseases», «Physical injuries sustained during training, and Rehabilitation», «Diets and Dietary Regimes», «Drinks, Food and Supplements Ingredients». These microfields are an integral part of fitness terminology, characterise it as a separate terminology system and are evidence of its structural and logical integrity.

The professional language of fitness is formed at the junction of sports, medicine and nutrition. The basis of the professional language of fitness is sports, medical and food terminology, as evidenced by the large number of borrowed terms from these fields of knowledge. However, in the professional language of fitness there are new terms that reflect the specifics of fitness in relation to other industries. These terms are new, created deliberately to denote new concepts. 
Among the terms borrowed from the above areas of the fitness system, there are terms of sports (e.g. competition, coach, exercise); medical terms and vocabulary of related disciplines (lactic acid, blood pressure, anemia); terms of nutrition (food quality, protein supplement, Ditch Dieting). Within the term fitness system you can find commonly used words (team, wellness, rest).

Structurally, the terms of the professional language of fitness are represented by terms-words, such as flexibility, endurance, eustress and terms-phrases, such as Graded Exercise Test, Resting Metabolic Rate etc. Word terms are represented by nouns, verbs and adjectives. The core of the professional language of fitness are noun terms (74\%), for example: activity, nutrition, diet; adjectives (16\%), such as enduring, regular and verbs $(9 \%)$, such as tone up, exercising.

One of the productive types of morphological word formation of the terminological system of fitness is suffixation. Characteristic of fitness terminology are models with suffixes: -tion, -ine, -er, -or, -ing, - ity, -ic, -al, -ed, -able, -ise, for example: prioritise, obesity, healthier, horizontal, production, satisfying.

Prefix derivatives of the English fitness terminology are formed with the help of prefixes and prefixoids. The most common among them are: over-, hyper-, re-, under-, non-, in-, bi-, anti-, hypo-, dis-, un-, for example: overeat, incorporating, intake, unhealthy, discomfort.

Among the most productive models of the formation of two-component terms in the professional language of fitness are models $\mathrm{N}+\mathrm{N}(48 \%$, strength training, fitness equipment), $\mathrm{A}+\mathrm{N}(42 \%$, glycemic index $)$. Among the threecomponent terms, the most productive models are $\mathrm{A}+\mathrm{N}+\mathrm{N}(28 \%$, concentric muscle action, effective circuit training $), \mathrm{N}+\mathrm{N}+\mathrm{N}(22 \%$, group fitness class $), \mathrm{A}+$ $\mathrm{A}+\mathrm{N}(9 \%)$. Within the professional language, there are four-component terms and five-component terms, for example: Excess Post-Exercise Oxygen Consumption, High Intensity Interval Training, slow twitch muscle fibers. The use of multicomponent formations is explained by the specifics of the syntactic organisation of the English language, due to which the kernel-noun shows the ability to multi-element definition.

The use of abbreviations is due to the tendency to language economy and the desire of the English language to monosyllabism. The following abbreviations have been identified, which is $3.78 \%$ of the total body of the terminology system such as BMI - Body Mass Index, DOMS --Delayed Onset Muscle Soreness, RPE - Rate of Perceived Exertion, HBOT - Hyperbaric Oxygen Therapy.

The professional language of fitness is also full of digital abbreviations: 1ATA (atmosphere absolute), 3SS (simple steps).

The initial type of abbreviations, which is the most common, nominates the main categories of concepts: types of exercise, physical processes in the body during or after training, diseases and syndromes, chemical compounds and drugs, standards and norms of consumption, food and sports supplements, physiological indicators. Structural and semantic analysis of the reduction of fitness terms allowed to identify such typical processes of their formation as truncation (professional $\rightarrow$ pro, pectorals $\rightarrow$ pecs) and telescopy (boxercise, aerobics).

At the heart of the process of semantic derivation is the productive metaphorisation of a commonly used word, for example, wind sprint - short-term acceleration while running, from the common word wind - has the ability to be gusty. 
Investigating the phenomenon of metaphorisation in the professional language of fitness, it should be noted that the leading role in creating the vocabulary of fitness is played by the similarity of realities between commonly used words and terms of fitness, which are reflected in the similarity of meanings. During metaphorical term formation, which is a process of secondary nomination, the newly created linguistic unit - the metaphorical term - tends to a kind of semantic development, as it begins to function in the newborn text as an interpreter of the new scientific concept. For example, the fitness term butterfly machine - a simulator, during the exercise the movements on it resemble a butterfly was formed by metaphorical transfer from the common word butterfly «butterfly». This example shows that there is a close and inextricable link between metaphorical processes and meaning abstraction.

The study of the scope of functioning of terms that have acquired new meanings through metaphorisation, allows us to identify two groups of such terms that are subject to transterminologisation by metaphorisation:

1) terms that have passed the stage of determinologisation and simultaneously function in the original terminological field without changes;

2) terms that are not common in common language.

In the first case, the metaphor connects the lexical units of two languages (professional language and common language), in the second - the lexical units of one language (professional language).

In order to identify metaphorical bases for transference and metaphorical models that function in the professional language of fitness, we conducted a study of terminological vocabulary, the source was the blogs of fitness trainers, namely Elle Linton «Keep it simpElle», Steph Gaudreau «Health from the inside out», Nicole Pearce "Pumps and Iron", Ben Greenfield BenGreenfieldFitness, Katie Dunlop "Love Sweet Fitness" and others. As a result of the study, metaphorical formations were found between the terms of fitness, which are based on the similarity of a feature of concepts or the similarity of several features:

1) Metaphor for the similarity of signs: triangle pose - standing pose is great for stretching the hips and lengthening the inner thighs, from triangle - is a closed figure with three sides.

2) Metaphor for resemblance to animals: butterfly crunch - lay on back with legs spread (feet together) in the butterfly position, crunch up to the left, center, to the right, back to center, from butterfly - insect;

3) Metaphor for the similarity of the name of the place: peak - the highest point of physical fitness, from peak - the top of the mountain

4) Metaphor for functional similarity: burn - burns more calories during intense training, from burn - to consume fuel and give off heat, light, and gases.

While metaphorical transference occurs in language through associations of similarity, metonymic transference - due to the adjacency of objects or concepts temporal, spatial, logical, causal, material, and others. Metonymy reflects the constant contacts between objects and is typed. The connection between the links goes from one word to another sequentially and indirectly, which radically distinguishes metonymy from metaphor.

Metonymic transfer is based on the relationship between process and result, process and person, process and object, part and whole, object and person (nutrition - nutrition and the science of nutrition; Due to the specialization of 
commonly used words, their semantics change, and they are used in the terminology of fitness to denote professional concepts.

In the professional language of fitness we distinguish the following models of metonymic transference:

1) Metonymic process - subject transfer: treadmill - monotonous mechanical work and a treadmill.

2) Metonymic subject - person transfer: ace - a team's best starting pitcher; also used as a verb, meaning "did very well" and ace - a playing card.

3) Metonymic process - result transfer: practice - training and exercise;

4) Metonymic result - process transfer: lunge - lunge and an exercise in which you step forward with one leg and bend your knees;

5) Metonymic process - person transfer: coach - one who instructs players in the fundamentals of a sport and directs team strategy and to coach.

It should be noted that metaphorical transfer in quantitative terms is more common than metonymic. To summarise, the term combines different types of meanings i. e. abstract and concrete, just as the metonymic connections of the meanings of the term are similar to the real relationships between objects, processes, phenomena of the objective world.

The development of polysemantic connections in the terminological system of fitness is established due to which the content of a language sign develops more intensively than its form. Significantly common in the term fitness system terms with two meanings, for example: squat - a squatting position and to position yourself close to the ground balancing on the front part of your feet with your legs bent under your body; deadlift - weightlifting competitions and lifting a barbell from the floor.

As a result of the destruction of the semantic connection between the meanings of one word, polysemy disintegrates and homonymy is formed. The terminology of fitness is characterised by the fact that its homonymous terms operate in different terminological systems and have different definitions. The most common is intersectoral terminological homonymy, for example:

- ABS (economic) - asset-backed securities are financial securities backed by assets such as credit card receivables, home equity loans, and auto loans.

- ABS (fitness) - abdominal back spine; this kind of fitness involves the muscles of the back and abdominals.

- ABS (technical) - an anti-lock braking system is a safety anti-skid braking system used on aircraft and on land vehicles;

- Game (sport) - is an activity or sport usually involving skill, knowledge, or chance, in which you follow fixed rules and try to win,

- Game (hunting) - wild animals that hunt for food.

- Internal homonymy within fitness occurs as follows:

- Scissors (fitness) - is an abdominal exercise that strengthens the transverse abdominals, helping flatten your belly and strengthen your entire core.

- Scissors (gymnastics) - a movement of the legs in a dance skill where the legs switch from split to other split.

The specificity of synonymous relations is due to the peculiarities of the lexical meaning of the term, which is limited to the content of the expressed professional concept. Examining the terminology of fitness, as a result of the analysis of the terms of professional language, it should be noted that the predominant synonyms are doublets: instruction - consultion; physical activity - exercise. 
Antonyms in the term fitness system are represented by a sufficient number of terms. Antonyms serve as a means of constructing the basis of professional vocabulary, around which its basic concepts are formed. Antonyms assess the relevant facts of reality, as well as clearly express the evaluation process in contrasting form, for example: high-intensity - lower-intensity; reduce - increase; wellness - illness.

Antonymy serves as a means of constructing the basis of professional vocabulary, around which its basic concepts are formed, for example: fine motor skills, gross motor skills (general motor skills), activity, inactivity.

Polysemic, homonymous, synonymous and antonymous relations play a fundamentally important organising role in individual subsystems of fitness vocabulary. Polysemic relations in the terminological system indicate that with the development of fitness the meaning of some terms is clarified, and others due to the lack of formal means of expression expand their semantic scope. The analysis allowed to identify intra-industry, intersectoral and external system polysemy of fitness terms based on functional characteristics.

\section{Textual parameters of professional fitness language}

The professional text arises in a complex communicative situation, in which the author with his communicative goal, and hence the communicative strategy, as well as the addressee with the set behavior of expectation and, if necessary, with the intention to react, take part as decisive factors. Under professional communication L. Hoffmann [3, p. 595-617] understands externally or internally motivated or stimulated external and internal systems of knowledge aimed at a chain of professional events that change the system of knowledge of one specialist and group of specialists. The main components of professional communication are the producer, professional text and its recipient. Professional text is the main tool allowing to fully disclose the features of professional communication.

According to the target audience, all professional texts are divided into:

1. Professional texts written by specialists, journalists-scientists, people who have a certain relation to the field, within which they write. The result of this activity is popular science literature in the form of books, non-fiction articles, etc. Target audience - diverse, starting from high school students to people with higher education. Schematically, this can be represented as follows: SPECIALIST / JOURNALIST-SCIENTIST $\rightarrow$ NON-SPECIALIST / AMATEUR.

2. Professional texts written by experts, specialists in the field for college or university students, future specialists in the field. Schematically, this can be represented as: SPECIALIST $\rightarrow$ FUTURE SPECIALIST. The result of such activities - textbooks, manuals, reference books, dictionaries, encyclopedias.

3. Fax texts written by researchers, specialists for scientists, specialists, graduate students: SCIENTIST SPECIALIST $\rightarrow$ SPECIALIST / SCIENTIST. These include scientific articles, monographs, dissertations, and industry encyclopedias.

To the text type SPECIALIST / JOURNALIST-SCIENTIST $\rightarrow$ NONSPECIALIST / AMATEUR text type we include popular science texts, which are a special field of analysis of the processes of representation of special concepts. In them, textual strategies are aimed at popularising the achievements of scientific 
thought and the implementation of appropriate communicative and pragmatic tasks, they are accompanied by the need to find the best means to disseminate scientific information. That is, popular science texts are created to promote certain achievements of human activity. The author presents the material taking into account the level of professionalism of the reader who is not a specialist in this field. This text type is characterised by elements of expressiveness, emotionality and subjective evaluation, which distinguish it from other types of texts.

The structural indicators of the terms also depend on the level of professionalism of the text and the communicative intentions of the producer. All professional fitness texts use graphic, lexical and syntactic tools to connect the text. For example: I've also successfully coached hundreds of men and women, with all different body types, on how to turn their own bodies into metabolically efficient fatburning machines, but now approach things from a more metabolically healthy standpoint, rather than a "nuke fat at all costs" standpoint; find that "WHY" for weight loss, and remind yourself of it every single day; If you aren't drinking enough water, it is likely contributing to your bloated belly; When too much fructose floods your body, your body stores it as fat. Furthermore, this leads to an excess of leptin; when one has too much leptin it's possible to become leptin resistant, meaning your body no longer can tell if you're full or not-and you keep eating and gaining weight.

The peculiarity of professional texts is manifested in the frequency of application of certain syntactic constructions. The leading role in the professional texts of fitness is played by simple common sentences, which promote the effective perception of the text by the recipient, activate cognitive activity and pragmatic expectations of readers, for example: People come in all shapes in sizes, depending on both your genetics and lifestyle choices.

The role of exclamatory and interrogative sentences, modalities and infinitive constructions is levelled with the growth of the professionalism of the text. The structural indicators of the terms also depend on the level of professionalism of the text and the communicative intentions of the producer.

\section{Conclusions}

The professional language of fitness is a relatively young professional language, which consists of a set of all language tools used in professional communication.

The concept of "fitness" has become so comprehensive that it has acquired, in addition to a purely professional interpretation, also social and philosophical significance. From a philosophical point of view, fitness can be described as a system of balance of physical, mental, social and intellectual state of modern man, who lives in harmony with the environment.

The professional language of fitness is formed at the junction of sports, medicine and nutrition. The basis of the professional language of fitness is sports, medical and food terminology, as evidenced by the large number of borrowed terms from these fields of knowledge.

The professional language of fitness consists of a set of all language tools used in it. Thematically, it includes subsystems of individual industries. The vocabulary of fitness is understood as the set of tokens of commonly used language, 
proper terms, terminological units of sports, medicine, nutrition, general scientific terms, nomenclature units, professionalisms used in professional communication.

Borrowing terms into the English professional language of fitness from various fields and creating your own terms indicates that fitness is a new field of knowledge, the terminology of which is organised into a coherent system that has some independence based on combining different concepts of sports, medicine and nutrition. This composition of fitness vocabulary is due to the fact that professional communication takes place not only at the level of professionals, but also between ordinary people who want to improve the quality of life and well-being.

According to the formal structure, terminological units are divided into terminological words, terms-phrases and terms-abbreviations. Terms-words at the morphological level are mainly represented by nouns and a small number of adjectives and verbs.

Fitness terminology is also updated through metaphorisation and metonymisation, which contributes to the development of polysemy and homonymy. These processes cannot be avoided, as the terms operate according to the same language rules as commonly used words. The language of fitness is characterised by antonyms, which serve as a means of constructing the basis of its professional vocabulary. The intersection of synonymous and antonymous relations testifies to the systemic terminology of modern English-language fitness.

The study of the functioning of terms in texts has shown the relationship between the type of text, its communicative orientation and the peculiarities of the functioning of terminological units in it. This dependence is expressed in the degree of saturation of different types of texts with terminological units. The higher the level of professional knowledge of the recipient, the richer and more complex in structure are the terminological units in the text.

In the linguistic and structural design of fitness texts, various morphological, stylistic, syntactic, graphic and visual means are used, which ensure proper communication between the producer and the recipient.

Professional texts are written by specialists, journalists-scientists, people who have a certain relation to the field, within which they write. The result of this activity is popular science literature in the form of books, non-fiction articles, etc. Target audience - diverse, starting from high school students to people with higher education. Schematically, this can be represented as follows: SPECIALIST / JOURNALIST-SCIENTIST $\rightarrow$ NON-SPECIALIST / AMATEUR. Professional texts written by experts, specialists in the field for college or university students, future specialists in the field. Schematically, this can be represented as: SPECIALIST $\rightarrow$ FUTURE SPECIALIST. The result of such activities - textbooks, manuals, reference books, dictionaries, encyclopedias. Fax texts written by researchers, specialists for scientists, specialists, graduate students: SCIENTIST SPECIALIST $\rightarrow$ SPECIALIST / SCIENTIST. These include scientific articles, monographs, dissertations, and industry encyclopedias.

Thus, professional language exists on the basis of commonly used language as a means of professional communication of specialists, and professional text is the main representative of professional communication, which provides an opportunity to fully disclose its features. The semantic core of any professional language is a term - a linguistic sign that correlates with a certain concept of professional language and enters into a systemic relationship with other words within a closed system. 
A comprehensive study of the professional language of fitness creates prospects for a comprehensive study of the translation aspects of fitness terms and the continuation of work on the unification and standardisation of terminology, which will contribute to the creation of a dictionary of fitness terms.

\section{References}

Hoffmann Lothar. 1985. Kommunikationsmittel Fachsprache: Eine Einfuhrung. Tubungen: Gunter Naer.

Hoffmann Lothar. 1985. Kommunikationsmittel Fachsprache. Eine Einführung. Auflage. Tübingen: Gunter Narr Verlag.

Hoffmann Lothar. 1993. "Fachwissen und Fachkommunikation. Zur Dialektik von Systematik und Linearität in den Fachsprachen“. Bungarten, Frachsprachentheorie, 595-617.

Felber, Helmut. 1984. "Terminology Manual”. General Information Programme and UNISIST. International Information Centre for Terminology. Paris: Unesco; Infoterm.

Leitchik, Vladimir and Shelov, Sergei. 2003. "Some Basic Concepts of Terminology: Traditions and Innovations". Journal of the International Institute for Terminology Research, 14: 86-101.

Leichik, Vladimir. 2007. Study of terms: subject, method, structure.

Smith, Aaron C. T. and Stewart, Bob 2012. "Body Perception and health Behaviours in an Online Bodybuilding Community". Qualitative Health Research, 22(7).

Smith Maguire, J. 2008. "Fit for Consumption". Sociology and the business of fitness. London; New York: Routledge.

Steen-Johnsen, Kari. 2007. "Globalized Fitness in the Norwegian Context". International Review for the Sociology of Sport, 42(3).

Stern, Marc. 2011. Real or Rogue Charity? Private Health Clubs vs. the YMCA, 19702010. Business and Economic History. 